\title{
Parâmetros para análise de carqueja: comparação entre quatro espécies de Baccharis spp. (Asteraceae)
}

\author{
Budel, J.M.*; Duarte, M.R.; Santos, C.A.M. \\ Departamento de Farmácia, Laboratório de Farmacognosia, \\ Universidade Federal do Paraná, Curitiba, PR
}

Recebido para publicação em: 18/09/2003 Aceito para publicação em: 26/01/2004

\begin{abstract}
RESUMO: Diferentes espécies de Baccharis são conhecidas popularmente como carqueja e empregadas indistintamente na medicina tradicional como estomáquicas e diuréticas. $O$ presente trabalho teve como objetivo contribuir para a determinação de parâmetros para o controle de qualidade e aplicação na indústria de fitoterápicos, através da análise de quatro espécies de carqueja: Baccharis dracunculifolia DC., B. articulata (Lam.) Pers., B. cylindrica (Less.) DC. e B. gaudichaudiana DC. De um modo geral, B. dracunculifolia apresentou os maiores teores de umidade e de perda por dessecação, enquanto que $B$. articulata mostrou os maiores índices afrosimétrico e de amargor e teores de extratos secos a frio e a quente e de cinzas totais. $B$. dracunculifolia foi a espécie que apresentou maior rendimento do óleo volátil. Alguns componentes do óleo volátil ocorrem nas quatro espécies, porém cada uma delas apresentou um perfil cromatográfico característico.
\end{abstract}

Unitermos: Baccharis dracunculifolia; Baccharis articulata; Baccharis cylindrica; Baccharis gaudichaudiana; Asteraceae; Carqueja; controle de qualidade.

ABSTRACT: Parameters for carqueja analysis: comparison among four species of Baccharis spp. (Asteraceae). Different species of Baccharis are commonly known as carqueja and are used indistinctly in the traditional medicine as stomachic and diuretic. This research aimed to carry out the drug analysis of Baccharis dracunculifolia DC., B. articulata (Lam.) Pers., B. cylindrica (Less.) DC. and B. gaudichaudiana DC. In general, B. dracunculifolia showed the highest results of water content, essential oil and loss on drying, while $B$. articulata showed the highest foam and bitter indices, ash and water soluble extractive results. Some components of the essential oil appeared in the four species, although each one showed a charateristic chromatographic profile by TLC analysis.

Key words: Baccharis dracunculifolia; Baccharis articulata; Baccharis cylindrica; Baccharis gaudichaudiana; Asteraceae; Carqueja; quality control. 


\section{INTRODUÇÃO}

A família Asteraceae apresenta um grande número de espécies, que são utilizadas como medicinais e a presença de várias classes de metabólitos secundários faz considerar que a composição química é mais importante do que a morfologia na evolução dessa família (OLIVEIRA et al., 1984; EMERENCIANO et al., 1986; CRONQUIST, 1988). Baccharis é um importante gênero desta família e compreende muitas espécies denominadas popularmente de carqueja e indicadas para o tratamento de distúrbios do sistema digestivo (SILVA JÚNIOR, 1997; MORS et al., 2000). Para o gênero existem relatos da presença de flavonóides (BIANCHI et al., 1993; GIANELLO et al., 2000; SHARP et al., 2001), diterpenos (FULLAS, 1991; GIANELLO et al., 2000), taninos (SÁ; NEVES, 1996; ORTINS; AKISUE, 2000), óleo essencial (FERRACINI et al., 1995; LAWRENCE; COMPANY, 1999; ORTINS; AKISUE, 2000; FRIZZO et al., 2001) e saponinas (BIANCHI, 1993; ORTINS; AKISUE, 2000). No Brasil, a carqueja está entre as dez plantas medicinais mais comercializadas (SILVA JÚNIOR, 1997) e o Paraná se destaca como seu maior produtor (CORREA JÚNIOR et al., 1991).

De acordo com Corrêa (1984), o nome carqueja deve ter sido dado primeiramente à espécie B. genistifolia DC. , mas depois foi se estendendo às outras espécies de ramos alados. Hoje, esse nome abrange até mesmo espécies de ramos não alados, como aquela conhecida como carqueja-do-pântano, que pertence ao gênero Hydrolea. As espécies conhecidas como carqueja são: B. articulata (Lam.) Persoon, B. dracunculifolia DC., B. trimera (Less.) DC. (SILVA JÚNIOR, 1997), B. cylindrica (Less.) DC., B. fastigiata Baker, B. gaudichaudiana DC., B. genistifolia DC., B. glaziovii Baker, B. junciformis DC., B. lundii DC., B. microcephala Baker, B. notosergila Griseb., B. opuntioides Mart., B. pauciflosculosa DC., B. pentaptera DC., B. polyptera DC., B. sagittalis (Less.) DC. e B. stenocephala Baker (BARROSO, 1976; CORRÊA, 1984).

O gênero Baccharis é uma rica fonte de óleo essencial utilizado na indústria de perfumaria (SILVA JÚNIOR, 1997). Vários trabalhos se detêm no teor de óleo encontrado em diversas espécies de Baccharis, como por exemplo, $0,3 \%$ para $B$. retusa (SILVA; GROTTA, 1971); 0,3\% para $B$. trimera (SIQUEIRA et al., 1986; FARMACOPÉIA, 2003); <0,2\% para B. myrtilloides e B. rufescens (ZUNINO et al., 1998); 0,16 a 0,3\% para $B$. salicifolia; 0,18 a 0,45\% para $B$. latifolia (LOAYZA et al., 1995) e 1,8\% para B. stenocephala (BONA et al., 2002).

A falta de parâmetros para identificação da matéria-prima vegetal causa grande confusão, impossibilitando seu controle de qualidade e a obtenção do efeito a que se propõe. Por essa razão, objetivou-se realizar métodos de análise farmacognóstica de $B$. articulata, $B$. cylindrica, $B$. dracunculifolia e $B$. gaudichaudiana, com a finalidade de demonstrar variações dos parâmetros analisados dessas quatro espécies.

\section{MATERIAL E MÉTODOS}

\section{Material vegetal}

Foram coletadas partes aéreas floridas das quatro espécies de carqueja, todas no Estado do Paraná, nas seguintes localidades: $B$. dracunculifolia DC., na região de Vila Velha, Ponta Grossa, em fevereiro de 2002; B. articulata (Lam.) Pers., em Campo Magro, em setembro de 2001; $B$. cylindrica (Less.) DC., em Campo Largo, em fevereiro de 2002; e B. gaudichaudiana DC., em Inácio Martins, em outubro de 2001. Com os exemplares foram montadas as exsicatas, identificadas por especialistas e os representantes equivalentes estão registrados no Herbário do Instituto de Ciências Naturais da UFRGS, sob os seguintes números: $B$. dracunculifolia - ICN n. 122946, $B$. articulata - ICN n.122945, B. cylindrica-ICN n. 122944 e B. gaudichaudiana-ICN n. 122943.

Rev. Bras. Farmacogn., V. 14, n. 1, jan.-jun. 2004. 


\section{Métodos de análise farmacognóstica}

As partes aéreas floridas de $B$. dracunculifolia, $B$. articulata e $B$. cylindrica foram secas em estufa a $40^{\circ} \mathrm{C}$ e fragmentadas no laboratório, enquanto que as partes aéreas floridas de $B$. gaudichaudiana foram secas e fragmentadas pelo produtor da planta.

Foram realizados os seguintes ensaios: determinação dos teores dos extratos aquosos secos a frio e a quente, de cinzas totais, de cinzas insolúveis em ácido, de umidade pelo método azeotrópico (WHO, 1998), índice afrosimétrico (FARMACOPÉIA BRASILEIRA, 1977), perda por dessecação por infravermelho (FARMACOPÉIA BRASILEIRA, 1988) e índice de amargor (FARMACOPÉIA BRASILEIRA, 1959; 2000). Os óleos essenciais das quatro espécies foram obtidos por hidrodestilação, em aparelho de Clevenger, para essências menos densas do que a água e analisados por cromatografia em camada delgada (CCD), com placas de gel de sílica $60\left(\mathrm{~F}_{254}\right)$ Merck® e a visualização foi feita com vanilina sulfúrica a 1\% (WAGNER; BLADT, 1996), seguido de aquecimento a $105^{\circ} \mathrm{C}$ por $10 \mathrm{~min}$.

Os resultados foram submetidos ao teste de rejeição de dados e expressos em valores médios das triplicatas (USP, 1975).

\section{RESULTADOS}

Os resultados das análises estão sumarizados nas Tabela 1 e Figura 1.

Tabela 1. Resultados da análise farmacognóstica de quatro espécies de carqueja.

\begin{tabular}{|c|c|c|c|c|c|c|c|c|c|c|}
\hline \multirow[b]{2}{*}{ ESPÉCIE } & \multicolumn{2}{|c|}{ Extrato seco } & \multirow[b]{2}{*}{$\begin{array}{c}\text { Índice } \\
\text { afrosimétrico }\end{array}$} & \multicolumn{2}{|c|}{ Teor de cinzas } & \multirow{2}{*}{$\begin{array}{c}\text { Teor de óleo } \\
\text { essencial } \\
(\mathrm{ml} / 100 \mathrm{~g})\end{array}$} & \multirow{2}{*}{$\begin{array}{l}\text { Teor de } \\
\text { umidade } \\
(\mathrm{ml} / 100 \mathrm{~g})\end{array}$} & \multirow{2}{*}{$\begin{array}{c}\text { Perda por } \\
\text { dessecação } \\
(\mathrm{ml} / 100 \mathrm{~g})\end{array}$} & \multicolumn{2}{|c|}{ Índice de amargor } \\
\hline & $\begin{array}{l}\text { A frio } \\
(\%)\end{array}$ & $\begin{array}{c}\text { A quente } \\
\text { (\%) }\end{array}$ & & $\begin{array}{l}\text { Totais } \\
\text { (\%) }\end{array}$ & $\begin{array}{l}\text { Insolúveis } \\
\text { em ácido } \\
\text { (\%) }\end{array}$ & & & & $\begin{array}{l}\text { Padrão } \\
\text { brucina }\end{array}$ & $\begin{array}{l}\text { Padrão } \\
\text { quinina }\end{array}$ \\
\hline $\begin{array}{l}\text { Baccharis } \\
\text { articulata }\end{array}$ & $19,7 \pm 2,1$ & $25,0 \pm 2,6$ & $22,0 \pm 2,9$ & $5,67 \pm 0,11$ & $0,84 \pm 0,02$ & $0,5 \pm 0,0$ & $8,1 \pm 0,6$ & $10,18 \pm 0,33$ & $25000 \pm 0$ & $230,0 \pm 0,0$ \\
\hline B. cylindrica & $11,7 \pm 2,5$ & $15,0 \pm 1,0$ & $10,0 \pm 0,0$ & $4,00 \pm 0,21$ & $1,06 \pm 0,14$ & $0,6 \pm 0,0$ & $8,0 \pm 0,4$ & $8,98 \pm 0,06$ & $16666 \pm 0$ & $153,3 \pm 0,0$ \\
\hline B. dracunculifolia & $15,0 \pm 1,0$ & $22,0 \pm 1,0$ & $10,0 \pm 0,0$ & $4,56 \pm 0,07$ & $0,75 \pm 0,11$ & $0,8 \pm 0,1$ & $10,9 \pm 1,4$ & $16,98 \pm 0,19$ & * & * \\
\hline B. gaudichaudiana & $13,0 \pm 0,0$ & $13,3 \pm 3,2$ & $13,0 \pm 0,0$ & $3,45 \pm 0,22$ & $0,91 \pm 0,20$ & $0,1 \pm 0,0$ & $4,7 \pm 0,8$ & $7,94 \pm 0,04$ & $3125 \pm 0$ & $28,8 \pm 0,0$ \\
\hline
\end{tabular}

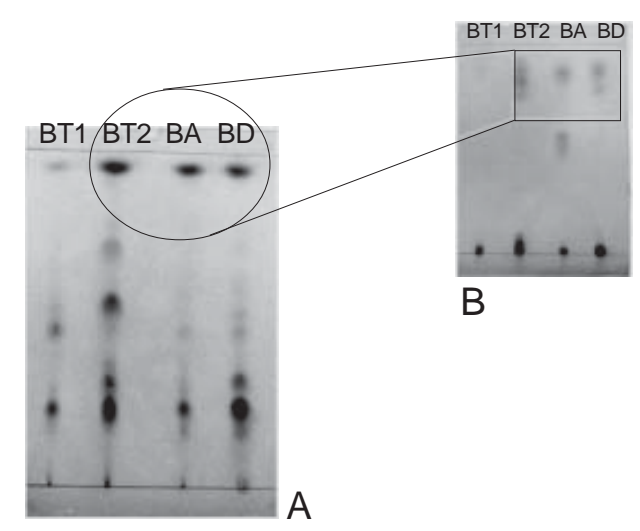

Figura 1. Cromatografia em camada delgada de amostras de Baccharis sp.: BTI (B. gaudichaudiana); BT2 (B. cylindrica); BA (B. articulata); BD (B. dracunculifolia). Fase móvel: $A$ - clorofórmio/benzeno (1:1); $\mathrm{B}-n$-hexano. 


\section{DISCUSSÃO}

Com relação aos dois métodos de determinação de extrato seco (a frio e a quente), $B$. articulata foi a espécie que apresentou maior quantidade de matéria extraível, 25\% e 19,7\%, respectivamente, enquanto que a menor quantidade obtida com o método a frio ocorreu para $B$. cylindrica $(11,7 \%)$ e com o método a quente para $B$. gaudichaudiana (13,3\%). A diferença dos teores médios entre os métodos a frio e a quente não foi expressiva para as quatro espécies de carqueja.

Ortins e Akisue (2000), utilizando os métodos de extração A e C da Farmacopéia Brasileira (1959) para $B$. articulata, determinaram o resíduo seco desses extratos encontrando valores médios de 14,72 e 12,67\%, respectivamente. Mello e Petrovick (2000) obtiveram 26,05\% de matéria extraível para B. trimera com o método preconizado pela WHO (1998). Esses resultados devem ser comparados com restrições, pois a metodologia aplicada por Ortins e Akisue (2000) foi diferente, e Mello e Petrovick (2000) não deixaram claro se foi utilizado o método a frio ou a quente.

Com base na informação de que algumas espécies do gênero Baccharis contêm saponinas, acreditou-se que o índice afrosimétrico poderia gerar parâmetros para o controle de qualidade. No entanto, dentre as quatro espécies estudadas, $B$. articulata foi a que apresentou o maior índice afrosimétrico (22), considerado pequeno se comparado com o de Polygala senega L., cujo alto teor de saponinas conduz à um índice afrosimétrico de 2500 (FARMACOPÉIA BRASILEIRA, 1959). Os resultados obtidos mostraram que esse índice não é adequado para avaliar a qualidade das quatro espécies estudadas.

Com relação às cinzas totais, $B$. articulata apresentou o maior teor (5,67\%), enquanto que $B$. gaudichaudiana, o menor (3,45\%). Quanto às cinzas insolúveis em ácido, a maior percentagem foi a de $B$. cylindrica (1,06\%), enquanto que a menor foi a de $B$. dracunculifolia $(0,75 \%)$. Para Ortins e Akisue (2000), B. articulata apresentou teores de cinzas insolúveis de 1,77\% e 0,9\%, nos extratos obtidos pelos processos A e C da Farmacopéia Brasileira (1959), respectivamente. Chicourel et al. (1997) avaliaram esse parâmetro físico-químico em função das estações do ano, segundo as normas analíticas do Instituto Adolfo Lutz; o teor de 1,6\% de cinzas foi encontrado para as três amostras estudadas, evidenciando que esse teor não se altera sazonalmente.

Não existem teores máximos para cinzas totais e insolúveis em ácido estabelecidos oficialmente para as espécies em estudo. Os teores de cinzas totais das quatro amostras são menores do que os de outras espécies farmacopéicas constituídas de partes aéreas, como Centella asiatica (L.) Urb. (11\%) e Malva sylvestris L. (16\%) (FARMACOPÉIA BRASILEIRA, 2000), Salvia officinalis L. (8 e 2\%) e Passiflora incarnata L. (11 e 3\%) (BRITISH PHARMACOPOEIA, 1996). Os baixos teores de cinzas insolúveis em ácido podem ser explicados pelos cuidados observados na obtenção dessas matérias-primas vegetais.

Plantas medicinais com forte sabor amargo são empregadas principalmente como digestivas, pois estimulam a produção de suco gástrico (MORS et al., 2000). Costa (1987) relatou a presença de sesquiterpenóides de propriedades amargas na família Asteraceae, e Zunino et al. (1998) afirmaram que a composição típica dos óleos voláteis de espécies de Baccharis, principalmente pela presença desses compostos, poderia ser utilizada como um critério químico para avaliar a qualidade em amargor.

B. articulata, conhecida como carqueja-doce, paradoxalmente, foi a espécie que apresentou os maiores índices de amargor: 25000 para o padrão brucina e 230 para o padrão quinina. 0 mesmo índice para o padrão brucina está indicado na monografia da espécie Gentiana lutea L., droga vegetal reconhecidamente amarga (FARMACOPÉIA BRASILEIRA, 1959).

A espécie $B$. cylindrica apresentou um índice de amargor relativamente alto, 16666 para o padrão brucina e 153,3 para o padrão quinina. B. trimera, conhecida como carqueja-amarga, apresentou um índice de 5000 para o padrão brucina (COSTA, 1987) e 31,3 para o padrão quinina

Rev. Bras. Farmacogn., V. 14, n. 1, jan.-jun. 2004. 
(MELLO; PETROVICK, 2000; FARMACOPÉIA BRASILEIRA, 2003). Para a espécie B. gaudichaudiana, foi encontrado um valor de 3125 pelo padrão brucina e 28,8 para o padrão quinina. Provavelmente, esse baixo índice é devido à presença de compostos doces e neutros, conforme relatado por Fullas et al. (1991). Esses autores testaram os gaudichaudiosídeos A-E quanto ao índice de dulçor. O gaudichaudiosídeo A exibiu potência de dulçor 55 vezes maior que uma solução de sacarose a $2 \%$, enquanto que o gaudichaudiosídeo $D$ foi totalmente amargo. Em $B$. dracunculifolia, não foi possível a percepção de sabor amargo nem nas soluções mais concentradas.

Para Wasicky et al. (1943) o índice de amargor tem bases teórica e experimental sólidas, o que o faz competir com os melhores métodos biológicos. Entretanto, Mello e Petrovick (2000) afirmaram que este teste pode apresentar erros devido à variabilidade individual, não reproduzindo estatisticamente informações reais. Assim, para realizar esse teste, é necessário um treinamento prévio do experimentador, que aliado à análise estatística, fazem com que a variabilidade biológica seja minimizada, tornando o método bastante confiável. Neste trabalho, observou-se que os valores são coerentes para os dois métodos e a presença de compostos doces, doce-amargos e amargos na espécie B. gaudichaudiana poderia também ocorrer nas demais espécies, dificultando a percepção do amargor.

Os componentes voláteis foram quantificados nas quatro amostras. De $B$. dracunculifolia foi obtido um rendimento de $0,8 \%$ de óleo essencial, enquanto que foi encontrado $0,25 \%$ por Siqueira et al. (1986); 0,16 a 0,32\% por Loayza et al. (1995) e 0,5 a 1\% por Weyerstahl et al., (1996). Para $B$. articulata, o rendimento foi de $0,5 \%$, sendo que outros trabalhos indicaram teores de $0,3 \%$ (SIQUEIRA et al., 1985 e 1986); 0,22\% (BONA et al., 2002) e < 0,2\% (ZUNINO et al., 1998).

B. dracunculifolia, B. articulata e B. cylindrica apresentaram bons rendimentos de óleo essencial, em parte devido à correta obtenção dessas drogas vegetais, destacando-se a coleta pouco antes da floração, quando o conteúdo de óleo deve ser máximo. B. gaudichaudiana apresentou um rendimento de óleo essencial muito baixo em relação às demais espécies. Essa matéria-prima foi preparada pelo produtor; provavelmente, a secagem foi feita em temperatura acima da adequada e associada à moagem a pó fino poderiam explicar o baixo rendimento encontrado.

O perfil cromatográfico permite, em muitos casos, uma confirmação da identidade de um óleo essencial e até a detecção de falsificações (FARIAS, 2000). Para as quatro espécies analisadas por CCD, a fase móvel que apresentou melhor perfil foi a mistura de clorofórmio:benzeno (1:1), porém foram observadas manchas concentradas na parte superior da placa cromatográfica, que foram melhor separadas quando se usou $n$-hexano. Alguns componentes do óleo essencial aparecem nas quatro espécies, especialmente os de $R_{f} 0,22,0,46$ e 0,95 (Figura 1-A). Um componente com $R_{f} 0,86$ também mostrou-se presente nas quatro espécies (Figura 1-B); porém, cada amostra apresentou um perfil cromatográfico característico para as duas fases móveis utilizadas.

Silva e Grotta (1971) realizaram uma análise por CCD do óleo essencial de B. retusa, utilizando como eluente o benzeno, e observaram a presença de quatorze componentes. Siqueira et al. (1985) verificaram a presença de oito manchas nos óleos voláteis de $B$. articulata e $B$. trimera, sendo particularmente notadas as que correspondem ao carquejol e acetato de carquejila, com valores de $R_{f}$ de 0,59 e 0,96, respectivamente, quando se utilizou como fase móvel clorofórmio.

Os teores médios de umidade encontrados para $B$. dracunculifolia, $B$. articulata e $B$. cylindrica foram considerados satisfatórios por situarem-se entre 8 e 14\%, como indicado na Farmacopéia Brasileira (1988). Esses resultados eram esperados pelas condições nas quais as drogas vegetais foram preparadas. Entretanto, B. gaudichaudiana apresentou um teor de umidade muito baixo, confirmando a suspeita de que essa espécie tenha sido seca em temperatura superior a $40 \stackrel{\circ}{ } \mathrm{C}$, o que resultou também em baixo rendimento do óleo essencial $(0,1 \%)$. 
Comparando-se o teor de umidade obtido pelo método azeotrópico e o resultado obtido pela perda por dessecação por infravermelho, observou-se que os valores desse segundo ensaio foram sempre superiores ao primeiro. Esse fato poderia ser explicado pelo fato de que na segunda metodologia ocorre a perda de outros constituintes químicos voláteis, como o óleo essencial, além da água.

\section{AGRADECIMENTOS}

Os autores agradecem aos taxonomistas Dra. I. J. M. Takeda (UEPG) e Dr. N. I. Matzenbacker (UFRGS) pela identificação das espécies, e ao Prof. P. V. Farago (UEPG) pela coleta das espécies.

\section{REFERÊNCIAS}

ALONSO, J.R. Tratado de fitomedicina - bases clínicas e farmacológicas. Buenos Aires: Isis, 1998.

BARROSO, G.M. Compositae - Subtribo Baccharidinae Hoffmann - Estudo das espécies ocorrentes no Brasil. Rodriguésia, v. 28, n. 40, p. 1-273, 1976.

BIANCHI, N.R.; SILVA, M.O; SPIASSI, L.; BERGONCI, J.I., MACHADO, C.A. Ensaio de toxicidade excessiva e screening fitoquímico de algumas espécies do gênero Baccharis L. (Asteraceae). Revista Brasileira de Farmácia, v. 74, n. 3, p. 79-80, 1993.

BONA, C.M.; BIASI, L.A.; NAKASHIMA, T.; ZANETTE, F.; CORRÊA JÚNIOR, C. Carqueja: Cultive esta idéia. Curitiba: SEAB-PR, 2002.

BRITISH Herbal Pharmacopoeia. 4. ed. Bournemouth: British Herbal Medicine Association, 1996.

CHICOUREL, E.L.; PIMENTA, D.S.; JORGE, L.I.F.; FERRO, V.O. Contribuição ao conhecimento analítico de três compostas medicinais. Revista Brasileira de Farmacognosia, v. 7/8, n. 1/2, p. 59-66, 1997.

CORRÊA, M.P. Dicionário das plantas úteis do Brasil e das exóticas cultivadas. Rio de Janeiro: IBDF, 1984. v.2. CORREA JÚNIOR, C; MING, L.C.; SCHEFFER, M.C. Cultivo de plantas medicinais, condimentares e aromáticas. Curitiba: Emater-Paraná, 1991.

COSTA, A.F. Farmacognosia. 2. ed. Lisboa: Calouste Gulbenkian, 1987. v. 3.

CRONQUIST, A. The evolution and classification of flowering plants. 2.ed. New York: New York Botanical Garden, 1988.

EMERENCIANO, V.P.; KAPLAN, M.A.C.; GOTTLIEB, O.R., BONFANTI, M.R.M.; FERREIRA, Z.S.; COMEGNO, L.M.A. Evolution of sesquiterpene lactones in Asteraceae. Biochemical Systematics and Ecology, v. 14, n. 6 , p. 585-589, 1986.

FARIAS, M.R. Avaliação de qualidade de matérias-primas vegetais. In: SIMÕES, C.M.O.; SCHENKEL, E.P.; GOSMANN,G; MELLO, J.C.P.; MENTZ, L.A.; PETROVICK, P.R. (Orgs.). Farmacognosia, da planta ao medicamento. 2.ed. Porto Alegre/Florianópolis: Ed. Universidade/Ed. da UFSC, 2000.

FARMACOPÉIA Brasileira. 3. ed. São Paulo: Andrei, 1977.

FARMACOPÉIA Brasileira. 4. ed. São Paulo: Atheneu, 1988.

FARMACOPÉIA Brasileira. 4. ed. São Paulo: Atheneu, 2000.

FARMACOPÉIA Brasileira. 4. ed. São Paulo: Atheneu, 2003.

FARMACOPÉIA dos Estados Unidos do Brasil. 2. ed. São Paulo: Siqueira, 1959.

FERRACINI, V.L.; PARAIBA, L.C.; LEITÃO FILHO, H.F.; SILVA, A.G.; NASCIMENTO, L.R.; MARSAIOLI, A.J. Essential oils of seven Brazilian Baccharis species. Journal of Essential Oil Research, v. 7, n. 4, p. 355 -367, 1995.

Rev. Bras. Farmacogn., V. 14, n. 1, jan.-jun. 2004. 
FRIZZO, C.D.; SERAFINI, L.A.; DELLACASSA, E., LORENZO, D.; MOYNA, P. Essential oil of Baccharis uncinella DC. from Southern Brazil. Flavour and Fragrance Journal, v. 16, n. 4, p. 286-288, 2001.

FULLAS, F.; HUSSAI, R.A.; BORDAS, E.; PEZZUTO, J.M.; SOERJARTO, D.D.; KINGHORN, A. D. Gaudichaudiosides A-E, five novel diterpene glycoside constituents from the sweet-tasting plant, Baccharis gaudichaudiana. Tetrahedron, v. 47, n. 40, p. 8515-8522, 1991.

GIANELLO, J.C.; CEÑAL, J.P.; GIORDANO, O.S.; TONN, C.E.; PETENATTI, M.E.; PETENATTI, E.M.; DEL VITTO, L.A. Medicamentos herbários en el centro-oeste argentino. II. "Carquejas": control de calidad de las drogas oficiales y sustituyentes. Acta Farmaceutica Bonaerense, v. 19, n. 2, p. 99-103, 2000.

LAVABRE, M. Aromaterapia: a cura pelos óleos essenciais. 4. ed. Rio de Janeiro: Record, 1997.

LAWRENCE, B.M.; COMPANY, R.J.R.T. Progress in essential oils. Perfumer \& Flavorist, v. 24, p. 53-63, 1999.

LOAYZA, I.; ABUJDER, D.; ARANDA, R.; JAKUPOVIC, J.; COLLIN, G.; DESLAURIERS, H.; JEAN, F. Essential oils of Baccharis salicifolia, B. latifolia and B. dracunculifolia. Phytochemistry, v. 38, n. 2, p.381389, 1995.

MELLO, J.C.P.; PETROVICK, P.R. Quality control of Baccharis trimera (Less.) DC.(Ateraceae) hidroalcoholic extracts. Acta Farmaceutica Bonaerense, v. 19, n. 3, p. 211-215, 2000.

MORS, W.B.; RIZZINI, C.T.; PEREIRA, N.A. Medicinal plants of Brazil. Michigan: Reference Publications, 2000.

OLIVEIRA, F.; AKISUE, G.; AKISUE, M.K. Farmacognosia. São Paulo: Atheneu, 1991.

OLIVEIRA, F.; ALVARENGA, A.M.; AKISUE, G.; AKISUE, M.K. Isolamento e identificação de componentes químicos de Mikania glomerata Sprengel e de Mikania leavigata Schultz Bip. ex Baker. Revista de Farmácia e Bioquímica da Universidade de São Paulo, v. 20, n. 2, p. 169-183, 1984.

ORTINS, G.M.M.; AKISUE, G. Estudo morfo-histológico, screening fitoquímico, constantes físicas e análise cromatográfica da droga e do extrato fluido, visando o controle de qualidade da espécie Baccharis articulata Pers. Lecta, v.18, n. 2, p.9-32, 2000.

SÁ, M.F.A.; NEVES, L. de J. Contribuição ao estudo das plantas medicinais Baccharis myriocephala DC. Revista Brasileira de Farmácia, v. 77, n. 3, p. 88-96, 1996.

SHARP, H.; BARTHOLOMEW, B.; BRIGHT, C.; LATIF, Z.; SARKER, S.D.; NASH, R.J. 6-Oxygenated flavones from Baccharis trinervis (Asteraceae). Biochemical Systematics and Ecology, v. 29, p. 105-107, 2001.

SILVA, J.B.; GROTTA, A.S. Anatomia da folha e óleo essencial de Baccharis retusa DC., Compositae. Revista de Farmácia e Bioquímica da Universidade de São Paulo, v. 9, n. 2, p. 321-326, 1971.

SILVA JÚNIOR, A.A. Plantas medicinais e aromáticas. Itajaí: Epagri, 1997. CD-ROM.

SIQUEIRA, N.C.S.; SILVA, G.A.A.B.; ALICE, C.B. Análise dos óleos essenciais de algumas plantas aromáticas tradicionais ou nativas no Rio Grande do Sul. Revista Brasileira de Farmácia, v. 67, p. 118-128, 1986.

SIQUEIRA, N.C.S.; SILVA, G.A.A.B.; ALICE, C.B.; NITSCHKE, M. Análise comparativa dos óleos essenciais de Baccharis articulata (Lam.) Pers. e Baccharis trimera (Less.) DC. (COMPOSITAE), espécies espontâneas no Rio Grande do Sul. Revista Brasileira de Farmácia, v. 66, p. 36-39, 1985.

UNITED State Pharmacopoeia. (USP) 19. ed. Rockville: United States Pharmacopoeial Convention, 1975.

WAGNER, H.; BLADT, S. Plant drug analysis: a thin layer chromatography atlas. 2.ed. Berlin: Springer, 1996.

WASICKY, R.; BARBIERI, E.; WEBER, H. Contribuição para o método de dosagem de princípios ativos em drogas e preparações pelo amargor. Anais da Faculdade de Farmácia e Odontologia da Universidade de São Paulo, v. 3, p. 113-119, 1943. 
WEYERSTAHL, P.; CHRISTIANSEN, C.; MARSCHALL, H. Constituents of Brazilian vassoura oil. Flavour and Fragrance Journal, v. 11, p. 15-23, 1996.

WORLD Health Organization (WHO). Quality control methods for medicinal plant materials. Geneva: WHO, 1998.

ZUNINO, M.P.; NEWTON, M.N.; MAESTRI, D.M.; ZYGADLO, J.A. Essential oils of three Baccharis species. Planta Medica, v. 64, n.1, p. 86-87, 1998.

* Autor para correspondência:

Profa. Dra. Jane Manfron Budel Universidade Federal do Paraná

Departamento de Farmácia

Rua Pref. Lothário Meissner, 3400

80210-170 - Curitiba - PR

E-mail: janemanfronb@uol.com.br 